\title{
EXTREMELY DELAYED CEREBRAL VASOSPASM AFTER SUBARACHNOID HEMORRHAGE
}

\author{
Angelo Daros Cecon', Eberval Gadelha Figueiredo², \\ Edson Bor-Seng-Shu ${ }^{3}$, Milberto Scaff ${ }^{4}$, Manoel Jacobsen Teixeira ${ }^{5}$
}

Delayed cerebral ischemia is an important cause of poor outcome in patients with aneurysmal subarachnoid hemorrhage (SAH). This clinical syndrome is caused by a critical reduction of cerebral blood flow brought on by a pathologic luminal narrowing of the cerebral arteries after SAH (vasospasm)'. It usually presents within 12 days after SAH and its pathogenesis probably depends upon inflammatory mechanisms. Its occurrence after 15 days is quite uncommon. Late diagnosis is often difficult and many times overlooked. Considering the role of triple $\mathrm{H}$ therapy in the treatment, differential diagnosis acquires paramount importance.
We present a rare case of extremely delayed cerebral vasospam after $\mathrm{SAH}$.

\section{CASE}

A 43 years-old female attended to the emergency room (ER) complaining of sudden, intense headache. Neurological examination disclosed meningismus. She was alert, presenting score 15 in the Glasgow Coma Scale. Head CT revealed a Fischer grade III SAH (Fig 1). Angiogram depicted an anterior communicating artery aneurysm, without signs of radiological vasoespasm (Fig 2). Aneurysm clipping was uneventfully performed three days after SAH, without need for temporary clipping. Postoperative
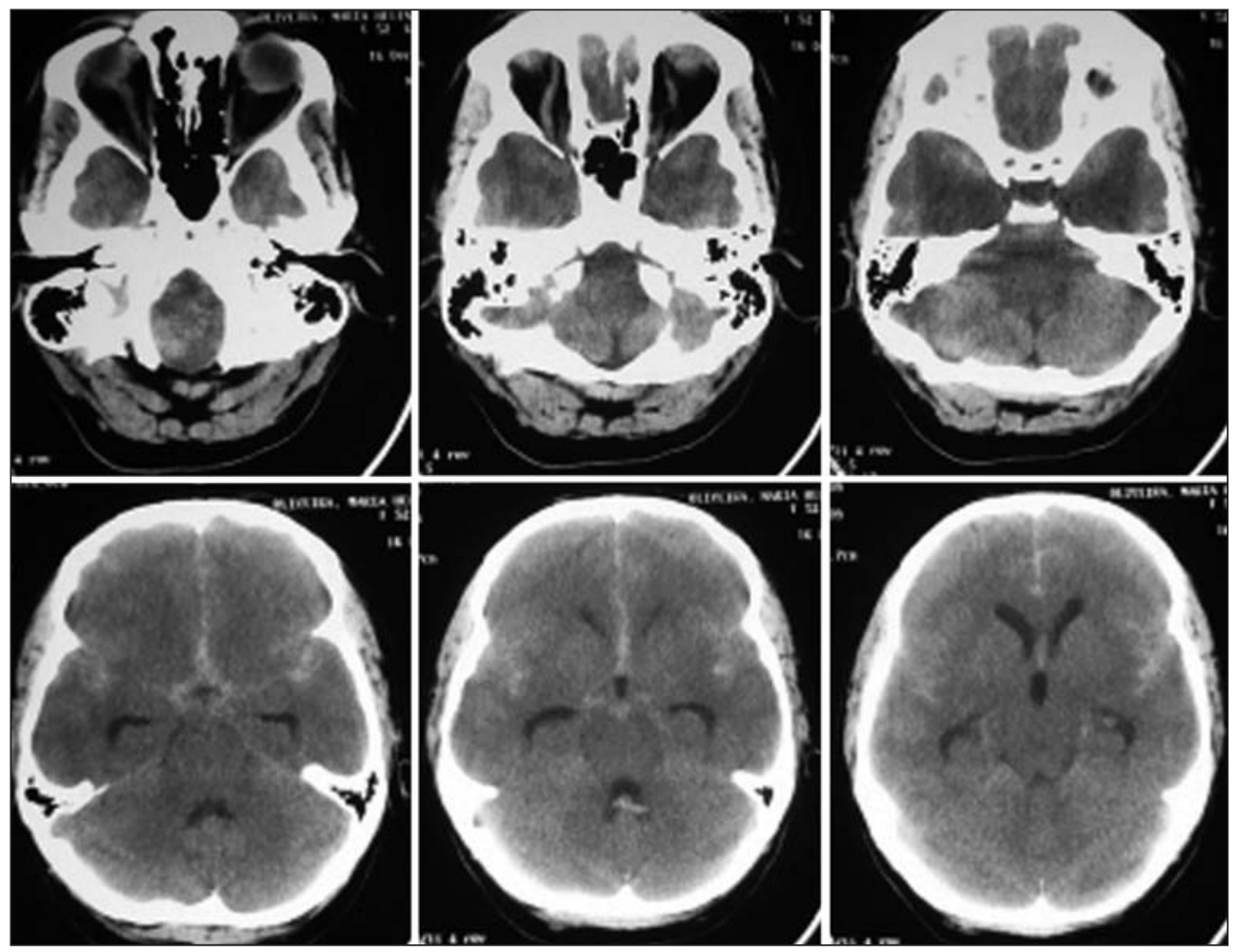

Fig 1. Axial head CT scan depicting a subarachnoid hemorrhage Fisher grade III.

\section{VASOESPASMO TARDIO APÓS HEMORRAGIA SUBARACNÓIDE}

'Resident, Division of Neurosurgery, University of Sao Paulo School of Medicine, São Paulo SP, Brazil (FMUSP); ${ }^{2}$ Coordinator, Cerebrovascular Surgical Team, Division of Neurosurgery, FMUSP; ${ }^{3}$ Coordinator, Hemodynamic Studies Center, Division of Neurosurgery, FMUSP; ${ }^{4}$ Professor and Chairman of

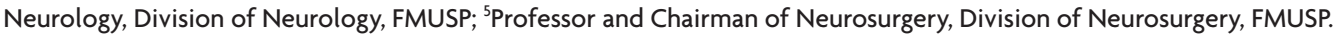

Received 8 February 2008, received in final form 28 April 2008. Accepted 24 May 2008.

Dr. Eberval Gadelha Figueiredo - Rua Oscar Freire 1456 / 34 - 05409-010 São Paulo SP - Brasil. E-mail: ebgadelha@yahoo.com 

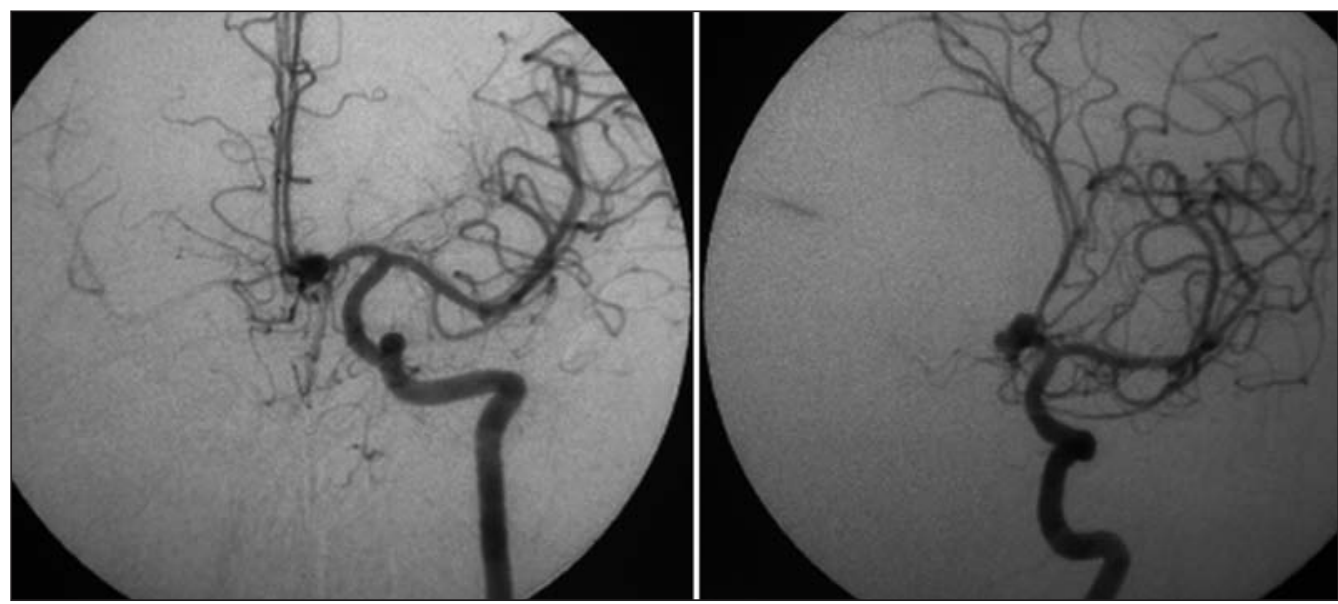

Fig 2. Left internal carotid artery angiogram showing an anterior communicating artery aneurysm.

CT scan did not reveal abnormalities. There was no clinical vasospasm during the first twenty-one days of $\mathrm{SAH}$, and patient was neurologically intact when discharged.

After 45 days, she returned to the ER presenting a left-sided hemiparesis. CT scan revealed a capsular ischemic area on the right cerebral hemisphere (Fig 3). No antecedents of hypertension and diabetes were observed. Arterial pressure levels were normal at admission. Serum levels of glucose and cholesterol were normal. Cardiac ultrasonography revealed no abnormalities as well as carotid and vertebral ultrasonography. Transcranial doppler (TCD) ultrasonography disclosed higher flow velocities suggestive of vasospasm. Triple $\mathrm{H}$ therapy was installed and patient completely recovered. Clinical improvement coincides with normalization of the TCD initial findings. Patient was discharged clinically intact.

\section{DISCUSSION}

Symptomatic vasospasm has been reported in $22 \%$ to $40 \%$ of patients suffering SAH and leads to high rates of morbidity $(34 \%)$ and mortality $(30 \%)^{2}$. The diagnosis of vasospasm is based upon clinical findings and evidence of luminal narrowing on angiogram or flow alterations on $\mathrm{TDU}^{3,4}$. Angiographic evidence of arterial spasm is seen in up to $70 \%$ of patients, and clinical manifestations are witnessed in 20 to $30 \%$ of patients ${ }^{5,6}$. Despite the introduction of nimodipine and the use of induced hypertension, symptomatic vasospasm still remains the major cause of morbidity and mortality in patients with $\mathrm{SAH}^{7-11}$.

Symptomatic vasospasm is defined by clinical criteria such as: 1) symptoms occurring between days 5 and 12 after $\mathrm{SAH}$, including headache, stiff neck, low-grade fever, insidious confusion, decline in level of consciousness and focal neurological deficit; 2) a head CT scan excluding other causes of worsening; and 3) no other identifiable cause of neurological deterioration ${ }^{8,9}$. Vasospasm can occur within 30 days after $\mathrm{SAH}$, and clinical vasospasm after

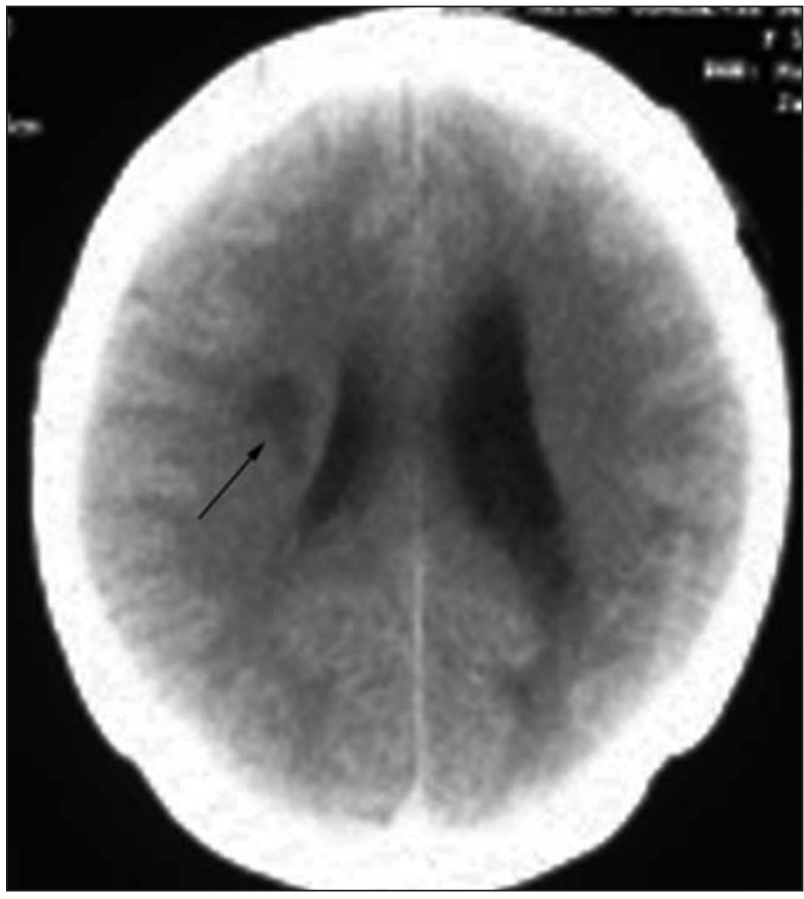

Fig 3. Capsular area of ischemia in the right cerebral hemisphere.

one month aneurysm bleeding is extremely rare and may difficult differential diagnosis. The case of patient who presented clinical deterioration after 45 days of SAH confirmed by TCD and clinical response to spasm therapy. In the present case, the age of the patient, absence of risk factors, normal carotid, vertebral and cardiac ultrasonography, associated with the TCD findings, coupled with the clinical improvement after triple $\mathrm{H}$ therapy and normalization of the TCD, practically excludes other causes of ischemic damage (embolism, aterothrombosis, etc) points to the vasospasm as the causal factor.

Delayed cerebral vasospasm, which is the most critical clinical complication that occurs after $\mathrm{SAH}$, seems to be 
associated with both impaired dilator and increased constrictor mechanisms in cerebral arteries ${ }^{3-8,10-13}$. Mechanisms contributing to development of vasospasm and abnormal reactivity of cerebral arteries after SAH have been intensively investigated in recent years ${ }^{10-13}$. The studies in the pathogenesis of vasospasm have focused on the inflammatory response after $\mathrm{SAH}$, which may play a relevant role in its development and maintenance ${ }^{3,4}$, secondary to a sudden abnormal contact of the extraluminal walls of arteries with all of the components of blood ${ }^{5}$. The inflammatory factors induced by SAH seem to be a great factor causing arterial spasm.

In the instance of SAH, a complex series of cellular and molecular events is elicited by the presence of blood clot in the subarachnoid space, culminating in a robust inflammatory response. Erythrocytes are dissolved, releasing oxyhemoglobin, which diffuses through the arterial wall of the endothelium ${ }^{13}$. A cascade of inflammation occurs, including infiltration and activation and recruitment of leukocytes, cytokine production, immunoglobulin and complement activation, and transcription factor activation, resulting in prolonged smooth muscle contraction and arterial spasm $^{13}$. These processes are auto-limited in the majority of the cases, however, delayed manifestations may occur.

It is possible that the mass effect of a large hematoma remaining in the sylvian fissure and an intracerebral hematoma after surgery may justify delayed development of spasm in some cases. Nonetheless, in the current case there was no hematoma left to explain late vasospasm. It is possible that the continuous inflammatory disease could bring to a progressive nip of brain arteries, and this was only revealed later in the course.
In conclusion, even though a very rare occurrence, extremely delayed vasospasm may occur after the first month after SAH. The neurological follow-up should be judicious and include frequent medical consultation in the first two months. Its occurrence must always be considered in the differential diagnosis of late neurological deterioration.

\section{REFERENCES}

1. Kassell NF, Sasaki T, Colohan ART, et al. Cerebral vasospasm following aneurismal subarachnoid hemorrhage. Stroke 1985;16:562-572.

2. Suarez J, Qureshi A, et al. Symptomatic vasospasm diagnosis after subarachnoid hemorrhage: evaluation of transcranial doppler ultrasound and cerebral angiography as related to compromised vascular distribution. Crit Care Med 2002;30:1348-1355.

3. Dumont AS, Dumont RJ, Chow MM, et al. Cerebral vasospasm after subarachnoid hemorrhage: putative role of inflammation. Neurosurgery 2003;53:123-135.

4. Sercombe R, Dinh YR, Gomis P. Cerebrovascular inflammation following subarachnoid hemorrhage. Jpn J Pharmacol 2002;88:227-249.

5. Schoch B, Regel J, et al. Analysis of intrathecal interleukin-6 as a potential predictive factor for vasospasm in subarachnoid hemorrhage. Neurosurgery 2007;60:828-836.

6. Weir BK, Macdonald RL, Stoodley M. Etiology of cerebral vasospasm. Acta Neurochir Suppl (Wien) 1999;72:27-46.

7. Mayberg MR, Batjer HH, Dacey RG Jr, et al. Guidelines for the management of aneurysmal subarachnoid hemorrhage: a statement for healthcare professionals from a special writing group of the Stroke Council, American Heart Association. Stroke 1994;25:2315-2328.

8. Haley EC Jr, Kassell NF, Torner JC. A randomized controlled trial of high-dose intravenous nicardipine in aneurysmal subarachnoid hemorrhage. Areport of the Cooperative Aneurysm Study. J Neurosurg 1993;78:537-547.

9. Ferguson S, Macdonald L. Predictors of cerebral infarction in patients with aneurysmal subarachnoid hemorrhage. Neurosurgery 2007; 60:658-667.

10. Ohman J, Servo A, Heiskanen O. Risk factors for cerebral infarction in goodgrade patients after aneurysmal subarachnoid hemorrhage and surgery: A prospective study. J Neurosurg 1991;74:14-20.

11. Sloan MA, Haley EC Jr, Kassell NF, et al. Sensitivity and specificity of transcranial doppler ultrasonography in the diagnosis of vasospasm following subarachnoid hemorrhage. Neurology 1989;39:1514-1518.

12. Zimmermann M, Seifert V. Endothelin and subarachnoid hemorrhage: an overview. Neurosurgery 1998;43:863-876.

13. Treggiari-Venzi M, Suter P, Romand JA. Review of medical prevention of vasospasm after aneurysmal subarachnoid hemorrhage: a problem of neurointensive care. Neurosurgery 2001;48:249-262. 